\title{
Analysis of Green Chemical Industry in Synthesis and Application of Polymer Materials
}

\author{
Bo Liu*
}

Yuanda Hengtong Material Co., Ltd. E-mail: liubo@sina.com

\begin{abstract}
With the continuous improvement of economic level and living standard in recent years, people's concept of environmental protection has become stronger and stronger. Developing green production in an all-round way will improve the contradiction between environmental protection and economic development. For the chemical industry, its production activities are a very critical link for the society, but at the same time, it is also the link that produces the most "three wastes". However, in the future development process of the chemical industry, green industry will be the most important development direction.
\end{abstract}

Keywords: Polymer Materials; Synthesis and Application; Green Chemical Industry

Since the reform and opening up, China's economy has developed rapidly. However, in the process of development, environmental problems have become increasingly prominent: extreme weather such as acid fog, smog and greenhouse effect have seriously affected people's normal life. Therefore, in order to realize the sustainable development of economy and ecological environment, China put forward the green development strategy of energy saving and emission reduction. Polymer synthetic materials are widely used in industrial production. Combining polymer synthetic materials with green strategy in application makes polymer synthetic materials gradually develop towards pollution-free and green environmental protection, thus realizing the development goal of green strategy.

\section{Understand polymer materials and characteristics}

Polymer material is a kind of polymer compound. Because of its different sources, polymer materials can be divided into many types, including natural polymer materials collected in nature, such as fiber, resin and rubber, and synthetic polymer materials, such as plastic and synthetic fiber. Different types of polymers have different advantages and disadvantages. The wear resistance and corrosion resistance of synthetic polymer materials are better than those of natural polymer materials. However, because of the high stability of synthetic polymer materials, it is difficult to deal with them. Because of the stable structure, it is not easy to be destroyed or corroded in nature, and exists in natural environment for a long time, which is a time bomb that causes pollution.

The basic characteristics of polymer materials are: it is non-toxic in the producing and catalytic process; the raw materials used are safe and, its application is convenient and harmless.

Green chemical technology, as a brand-new chemical technology, has achieved a breakthrough in the original chemical production technology, greatly improved

Copyright (C) 2020 Bo Liu

doi: 10.18282/ims.v3i2.350

This is an open-access article distributed under the terms of the Creative Commons Attribution Non-Commercial License

(http://creativecommons.org/licenses/by-nc/4.0/), which permits unrestricted non-commercial use, distribution, and reproduction in any medium,

provided the original work is properly cited. 
the comprehensive quality of chemical production, effectively controlled the cost, improved the comprehensive efficiency of chemical production, and made the whole production process gradually standardized and scientific. Modern enterprises vigorously develop green chemical technology, actively carry out the improvement and transformation of traditional chemical technology, make up for the previous technical deficiencies, and provide strong technical support for future chemical production. Developing green chemical industry can greatly reduce environmental pollution. Traditional chemical production consumes a lot of chemical raw materials, which causes a relatively large amount of pollutants in production, causing great harm to the surrounding environment. The development of green chemical industry has solved the problem of environmental pollution and gradually achieved the goal of environment-friendly industrial development.

At the same time, the development of green chemical industry can also greatly reduce the production cost of enterprises, and the consumption of chemical raw materials is relatively small, and most of the materials can be recycled repeatedly, which not only reduces the overall cost of chemical production, but also increases the comprehensive benefits of chemical production and provides technical support for green production and recycling production of chemical industry. Green chemical industry is very important for the development of chemical enterprises. Chemical enterprises continue to effectively promote the application of green chemical industry, gradually realize the transformation of modern chemical industry, expand the technological advantages of chemical production, and meet the development requirements of modern society. The comprehensive popularization and application of green chemical technology has adapted to the general trend of social development and is positive and beneficial.

\section{The green strategy for the syn- thesis and application of polymer materials}

\subsection{Non-toxicity of raw materials}

In today's research process of polymer chemical materials, the biodegradation technology is gradually introduced to ensure the non-toxic and green of polymer chemical materials, which is also a hot field of chemical research. Biodegradation of polymer chemical materials is widely used, including natural organic polymer materials and synthetic organic polymer materials. This technology is very practical for the research of starch, alginic acid, polyamino acid and other polymers. At present, many materials in the field of medicine are produced in this green and non-toxic form to achieve harmony and compatibility with human body.

\subsection{The synthesis of polymer raw materials is developing towards innocuity}

The synthesis of polymer raw materials is also developing towards green. In the process of chemical synthesis, many polymer chemical materials can be synthesized by one-step catalysis, and the conversion utilization rate can reach $100 \%$. Moreover, this process avoids the use of toxic chemical catalysts and changes the traditional operation mode. For example, the synthesis of adipic acid is to use biosynthetic technology to make its production process completely green, safe and operable. The traditional method for producing propylene oxide is a two-step reaction, and chlorine gas is used in the middle. This kind of gas has certain toxicity and will cause environmental pollution. But now, this production method has been changed at home and abroad, and the catalytic oxidation method is adopted to make full use of raw materials in the production reaction process, without producing some substances to pollute the environment. At present, in the process of making synthetic chemical materials, many of them are gradually improving the situation of toxic wastes or emissions produced by material synthesis, and developing towards the direction of green ecology and environmental protection.

\section{Greening of synthetic raw mate- rials}

Many living materials are made of raw materials synthesized by polymers. Especially medical materials, these materials must be non-toxic during use, and they must be biodegradable and acceptable to human immune system. Therefore, the requirements for synthetic raw materials must be green and safe. In recent years, many achievements have been made at home and abroad.

In 1988, there were related studies in Holland to study polylactic acid network elastomer materials, which 
were synthesized from green raw materials and could be biodegraded. They extended the chain of star-shaped prepolymer formed by inositol, L-lactide and so on with lysine diisocyanate vinegar. LDI can be called "green" diisocyanate chain extender, because the final degradation products of LDI chain extender are ethanol, lysine, etc. These degradation products are non-toxic and can be fully utilized biologically. In the process of forming this polymer, not only is the final product environmentally friendly and safe, but its raw material inositol is one of vitamins needed by human body. Lactic acid, 6-alkyl caproic acid, etc. are quite common in biomedicine, and they are also some safe and "green” substances. It can be said that this process is close to "completely green”. In 1994, Strey and other scholars made further research on this basis, and synthesized LDI polylactic acid derivatives with this green reagent. With high crystalline polyglycolic acid fiber as reinforcing material, nontoxic and bioabsorbable orthopedic fixation composites were prepared.

\section{Green catalyst}

In the research process of polylactic acid materials, although the current polymer raw materials and polymers have been basically green and non-toxic, one factor may be overlooked in this process, that is, the use safety of catalysts. For example, stannous octoate is mostly used as an intermediate catalyst to accelerate the chemical reaction. However, this catalyst may be physiologically toxic because it contains tin salt, and it may cause poisoning if absorbed by human body. In comparison, it is safe and reliable to use biological enzyme as catalyst. The bottleneck of using biological enzymes for catalysis lies in the limited variety of enzymes, which leads to the lack of corresponding biological enzymes for catalysis in some chemical reactions. Among the current high molecular polymers, although the atomic utilization rate of some polyaddition reactions can reach $100 \%$, the impact of the use of various catalysts and additives on the safety situation cannot be ignored. Especially in medical articles, the safety of these materials must be tested and assessed. The development of green catalyst road is worth exploring further.

\section{Safe application of synthetic polymer materials}

Synthetic polymer materials may do some harm to the environment, and the problem of green and non-toxic should be considered in the garbage disposal of unusable polymer materials. The right method must be chosen to use these polymer materials safely.

Biodegradable polymer synthetic materials can be treated by landfill. Non-biodegradable polymer material wastes are classified into recyclable wastes and non-recyclable wastes. Recyclable polymer materials are classified and sorted to realize recycling and reduce the waste of resources. Incinerable polymer materials can be incinerated, and the heat energy released during garbage incineration can also be utilized.

(1) The environmentally inert polymer materials that can be recycled and recycled, such as PP, PE, PET, Nylon 66, PMMA, PS, etc., should be reused as much as possible, and the landfill method should be avoided as much as possible to treat the environmentally inert plastic waste.

(2) Polyolefins such as PP and PE have high calorific value, which is equivalent to fuel oil, and have harmless combustion characteristics. Therefore, the huge heat energy generated by the combustion of these polymer materials can be converted into electric energy or other forms of energy, thus avoiding the pollution of heat energy. At present, the key to the successful implementation of transforming energy from municipal solid waste is to remove PVC, avoid mixing with PP, PE, etc., and avoid wasting energy due to difficulty in energy recovery.

(3) PVC should be used reasonably. The manufacture, processing, use and waste disposal of PVC all involve environmental problems, among which the most dangerous is the disposal of PVC waste. There are many additives used in the processing of PVC, and improper use will cause toxic substances in the materials to seep out, so contact with food and medical products should be avoided as much as possible. Incineration should be avoided as far as possible in the treatment of PVC waste, because this polymer material will produce toxic substances in the process of incineration, which will cause great harm to the environment. PVC should be withdrawn from packaging, toys, plastic film and other application fields with short service life as soon as possible. At the same time, in view of the advantages of saving natural resources, wide applicability, low price, 
non-flammability, good blood compatibility, etc. More efforts should be made on the research on PVC production, processing, use and waste treatment.

\section{Conclusion}

To sum up, in recent years, many industries have applied the related concepts of green economy, especially in the context of the contradiction between the current ecological environment and economic development, and the application of green industry will effectively promote the improvement of environmental problems. Therefore, in recent years, green economy has been developed in many fields in China. Non-toxicity and greening in the synthesis and application of polymer materials will be the development trend of the chemical industry in the future. If the green production strategy in the synthesis and application of polymer materials can be utilized, the polymer synthetic materials can gradually show the characteristics of pollution-free and environmental protection, which will be more in line with the relevant concepts of sustainable development. However, as far as the current situation is concerned, there are still some problems in the synthesis and application of polymer materials, and more efforts need to be put into exploring, so that the synthesis of polymer materials can achieve a more ideal sustainable development process.

\section{References}

1. Wang M, Ma Y. Green strategy in the synthesis and application of polymer materials (in Chinese). China Chemical Trade 2019; (13): 115.

2. Song R, Li R. Application of new chemical materials from the perspective of environmental protection (in Chinese). China Metal Bulletin 2018; (12): $118+120$.

3. Liu A. Application of organic chemistry in the synthesis of polymer materials (in Chinese). Metallurgy and Materials 2018; (6): 13.

4. Li H. Application prospect of polymer chemical materials (in Chinese). China Chemical Trade 2018; (3): 252

5. Fu X. Application analysis of organic chemistry in polymer material synthesis (in Chinese). Chinese Information 2019; (8): 111. 